\title{
Experimental Histological Evidence of the Safety of Transcranial Direct Current Stimulation as a Therapeutic Procedure
}

\author{
Ogunnaike Philip Olubunmi ${ }^{1}$, Olatunji Sunday Yinka ${ }^{1,2, ~ *}$, Owolabi Joshua Oladele ${ }^{1}$, \\ Olanrewaju John Afees ${ }^{1}$, Aminu Azeezat Jolade ${ }^{1}$, Fabiyi Sunday Oluseyi ${ }^{1}$ \\ ${ }^{1}$ Department of Anatomy, Ben Carson Senior School of Medicine, Babcock University, Ilisan Remo, Nigeria \\ ${ }^{2}$ Department of Anatomy and Cell Biology, Faculty of Basic Medical Science, College of Health Sciences, Obafemi Awolowo University, \\ Ile-Ife, Nigeria
}

\author{
Email address: \\ olatunjis@babcock.edu.ng (O. S. Yinka) \\ ${ }^{*}$ Corresponding author
}

\section{To cite this article:}

Ogunnaike Philip Olubunmi, Olatunji Sunday Yinka, Owolabi Joshua Oladele, Olanrewaju John Afees, Aminu Azeezat Jolade, Fabiyi Sunday Oluseyi. Experimental Histological Evidence of the Safety of Transcranial Direct Current Stimulation as a Therapeutic Procedure. American Journal of Psychiatry and Neuroscience. Vol. 6, No. 3, 2018, pp. 78-85. doi: 10.11648/j.ajpn.20180603.15

Received: June 28, 2018; Accepted: August 1, 2018; Published: September 13, 2018

\begin{abstract}
Transcranial direct current stimulation (tDCS) is a type of electrical modulation of the nervous system activity which involves the uses of low current to stimulate specified areas of the brain using electrodes to the scalp. This study was carried out to investigate if tDCS which is being used in the treatment of various disorders of the brain could have any possible side effects that might be worse than the treated disorder or any effects of tDCS on the cytoarchitecture of the dorsolateral prefrontal cortex. A total of 32 adult male Wistar rats were used and were placed into 5 groups (A-E). Rats in group A were divided into two groups A (SHAM) (tDCS for 30seconds) and A (N-SHAM). Rats in groups B, C, D and E were stimulated for $5,10,15$, and 20 minutes with 12 Volt respectively for the duration of 14 days and the animals were euthanized on the last day of the experiment two hours post brain stimulation. The specimen were subjected to gross morphological analysis and basic demonstration of the DLPFC using $\mathrm{H} \& \mathrm{E}$ and special stains. There was no significant difference in the neuronal structure and the supporting cells of the brain across the groups A (SHAM), B (5MINS), C (10MINS), D (15MINS), E (20MINS) when compared with control group A (N-SHAM) which suggest that tDCS does not have any neurodegenerative effects and could be safe in its use as neuro-stimulator to enhance cognitive ability in healthy individuals.
\end{abstract}

Keywords: Transcranial, Stimulation, Dorsolateral, Prefrontal, Cytoarchitecture, Electrodes

\section{Introduction}

Transcranial direct current stimulation is a purposeful modulation of the nervous system's activity that involves the use of constant but yet low controlled current delivered to the brain area of interest via electrodes to the brain. Evidences show that $\mathrm{tDCS}$ was originally developed to aid the treatment of various neurological and psychological (mental) disorders such as major depressive disorder [1]. New experiments and researches show the possibility of the use of tDCS in the treatment of schizophrenia [2], memory deficits in Parkinson's and Alzheimer's disease [3]. There is a rapid growing use of tDCS by the general public to enhance cognitive ability.

There is little or almost no existent report on the effect of transcranial direct current stimulation on the cytoarchitecture of the brain which is the main purpose of this research. The extensive prospective use of modernized tDCS in the treatment of various disease raises the concern on the effect of tDCS on the brain as there could be possible side effects that might be worse than the treated disorder.

The dorsolateral prefrontal cortex (DLPFC) is also known as the Brodmann's area 46 which is between the Brodmann's area 10 and Brodmann's area 45, it occupies the middle frontal gyrus and the rostral portion of the inferior frontal 
gyrus, the area 46 is based on the cytoarchitecture rather than the function. It serves a highly important executive role within the brain and subsequently is the main site of cognitive control in both humans and monkeys [4]. The DLPFC has long been implicated in higher cognitive functions such as switching attention, working memory, maintaining abstract rules, and inhibiting inappropriate responses [5]. The DLPFC is also involved in the preparation of saccadic eye movements [6].

The layers of the cortex is the same in all division and it is the same in the DLPFC, the different cortical layers each contain a characteristic distribution of neuronal cell types and connections with other cortical and subcortical regions. There are 6 main layers from the outside (pial surface) to the inside (white matter) and they are the molecular layer, external granular layer, external pyramidal layer, internal granular layer, internal pyramidal layer and the polymorphic or multiform layer [7]. The DLPFC is composed mainly of spatial selective neurons which makes it have a neural circuitry that encompasses the entire range of sub-functions necessary to carry out an integrated response such as retention in short term memory, sensory input and motor response such as retention in short term memory, sensory input and motor signaling [8] this area of the brain undergoes prolonged development and maturation as it continues unto adulthood. The area is more functional than anatomical. The DLPFC is the highest area of the cortex that is involve in motor planning, organization and regulation [9]. The spatial selective neurons contained in the DLPFC ensures that the neural circuitry functions approximately in order to carry out integrated responses such as sensory input, retention in short term memory and motor signaling [8].

Transcranial direct current stimulation of the DLPFC results in a shift in the excitability which occur during and after stimulation [10]. Research in enhancing working memory is definitely one of the major things that can be done with the use of the tDCS. Anodal tDCS applied on the DLPFC facilitate working memory processes [11]. Findings show that anodal tDCS over the right DLPFC may prevent working memory impairment induced by acute stress [12].

Stimulation of the primary motor cortex (M1) and dorsolateral prefrontal cortex (DLPFC) reduces the perception of pain. Researches show that anodal tDCS of the DLPFC could decrease the perception of unpleasantness and reduces emotional discomfort or pain.

\section{Materials and Methods}

Thirty two (32) adult male Wistar rats with average weight of $150 \mathrm{~g}$ were used for this research. The animals were grouped into 5 (A, B, C, D and E) of 6 animals in groups B, $\mathrm{C}, \mathrm{D}$ and $\mathrm{E}$ except group A which was divided into A (sham) and $\mathrm{A}(\mathrm{N}$-sham) with four animals in each. Rats were housed in plastic cages and given food and water ad libitum. Group A (sham) was the sham control that was stimulated for 30seconds and group A (N-sham) were not given any stimulation. Groups B, C, D and E were electrically stimulated using the same volts but with different durations.

The transcranial direction current stimulation device used in the experiment was the Brain Stimulator tDCS travel model V2.0, which was ordered from the brain stimulator, based in California, United States of America. The electrodes were adapted to be suitable for the rat model.

GROUP A1 (sham): Control group was given a sham (stimulated for 30 seconds) for 14 days

GROUP A2 (N-sham): Control group which was not given any stimulation

GROUP B: Stimulated with $12 \mathrm{~V}$ for 5 minutes daily for the duration of 14 days

GROUP C: Stimulated with $12 \mathrm{~V}$ for 10 minutes daily for the duration of 14 days

GROUP D: Stimulated with $12 \mathrm{~V}$ for 15 minutes daily for the duration of 14 days

GROUP E: Stimulated with $12 \mathrm{~V}$ for 20 minutes daily for the duration of 14 days

Animal fur medial to the ears of each experimental animals was shaved off to double-check the electrode-scalp contact. Brain stimulator was used to stimulate the experimental animals' brain and the electrodes were placed on both sides, medial to the ears and were held in place with tape. The duration and voltage used in the present study were selected based on previous works [13-17] and also based on the pilot study that we conducted.

On the last day of the experiment, two hours after the experimental procedures, the animals were euthanized. The extracted tissues were fixed in $10 \%$ formol saline, and then processed 48hours later for routine histomorphological analysis. Hematoxylin and Eosin staining method was used to demonstrate the general histology and morphology of the dorsolateral prefrontal cortex of the control and the stimulated groups and Luxol Fast Blue and Bielschowsky stains were used to demonstrate the Nissl substance, neurofibrils and senile plagues respectively.

Photomicrographs of the H\&E stained slides were imported into Image $\mathrm{J}$ software for both the neuronal cell count and glia cell (supporting cells) count and the values obtained were evaluated by one way analysis of variance (ANOVA) using GraphPad Prism 6 (GraphPad Inc. San Diego US), this was followed by Student Newman-Keuls (SNK) test for multiple comparisons. A value of $\mathrm{p}<0.05$ was considered to indicate significant difference between groups.

\section{Results}

Figure 1 shows the photomicrograph of the $\mathrm{H} \& \mathrm{E}$ staining of the prefrontal cortex of the brain at X100 showing the 6 layers which are the molecular, external granular, pyramidal, inner granular, ganglionic and multiform layer of the cerebral cortex across all the groups. There was no significant difference between group A (N-SHAM) and A (SHAM). There was no observable difference across the groups B, C, $\mathrm{D}$ and $\mathrm{E}$ when compared to group A (N-SHAM) and between each other.

Figure 2 is the photomicrograph of the $\mathrm{H} \& \mathrm{E}$ staining of 
the prefrontal cortex of the brain at X400 showing identifiable neuron, neuropil and glial cells. There was no significant difference between the group A (N-SHAM) and group A (SHAM) in the general histology. There was no observable difference across the treated groups B (5MINS), C (10MINS), D (15MINS), and E (20MINS) when compared to group A (SHAM).

Figure 3 is the photomicrograph of the Luxol Fast Blue staining of the prefrontal cortex of the brain at X400 showing identifiable myelin sheath, neuron and neuropil. The structure of the myelin sheath, neuron and the neuropil in group A (N-SHAM) is not difference from that of group A (SHAM). There was no significant difference in the structure of the myelin sheath, neuron and neuropil when compared to
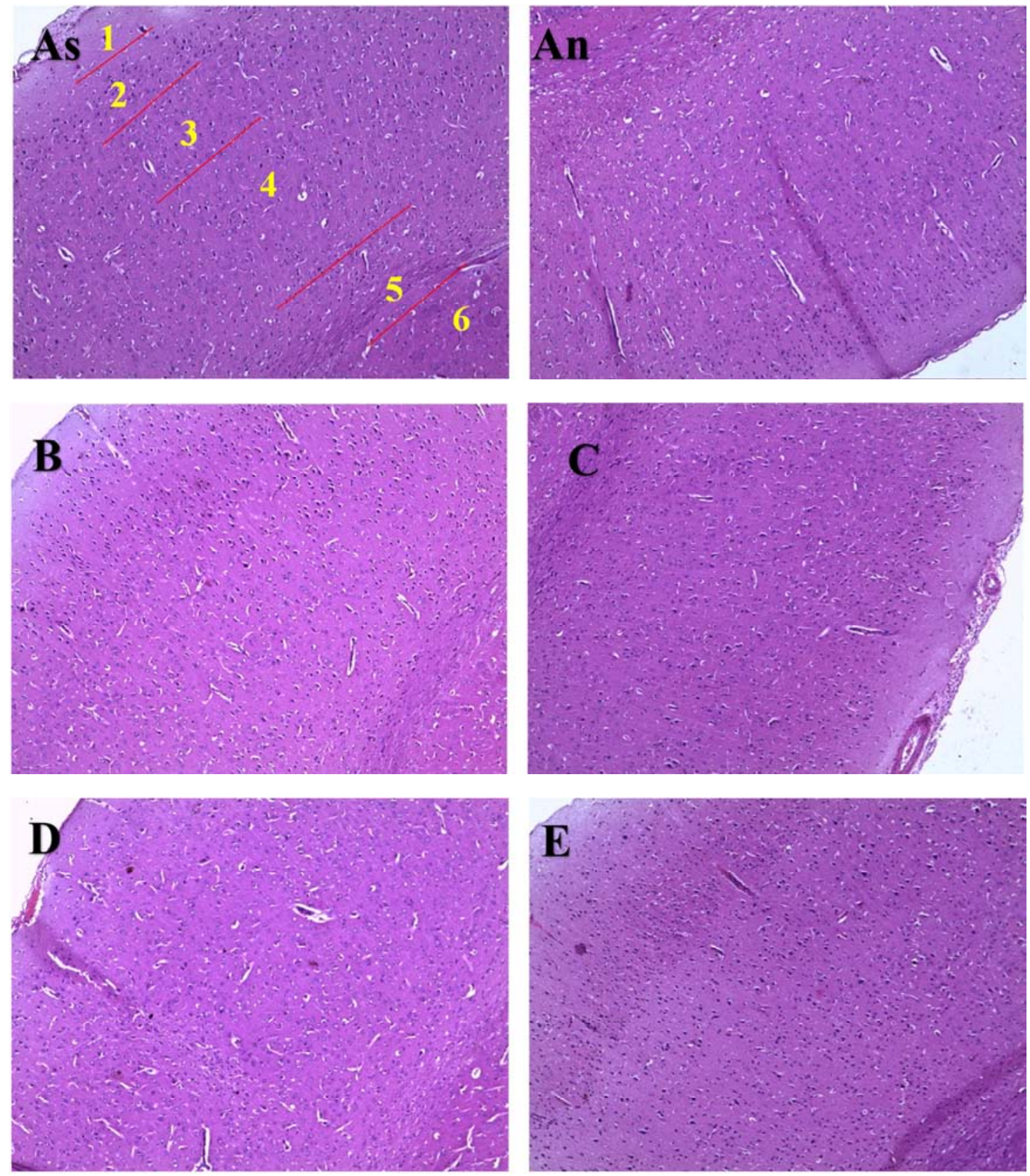

Figure 1. $H \&$ E stained prefrontal cortex (100X). As; group A (SHAM), An; group A (N-SHAM), B; group B (5MINS), C; group C (10MINS), D; group D

(15MINS), E; group E (20MINS). $1=$ molecular layer, $2=$ external granular layer, $3=$ pyramidal layer, $4=$ inner granular layer, $5=$ ganglionic layer, $6=$ multiform layer.

groups B (5MINS), C (10MINS), D (15MINS), and E (20MINS).

Figure 4 is the photomicrograph of the Bielchowsky's staining of the prefrontal cortex of the brain with identifiable nerve fibers (Axon and Dendrites), neuron and glial cells. There was no observable difference in the cell processes (Axon and Dendrites) of groups A (SHAM) when compared with group A (N-SHAM). The pyramidal cells are well displayed in the sections and there was no significant difference across all groups. There was no observable difference across all groups B (5MINS), C (10MINS), D (15MINS), and E (20MINS) when compared to group A (SHAM).
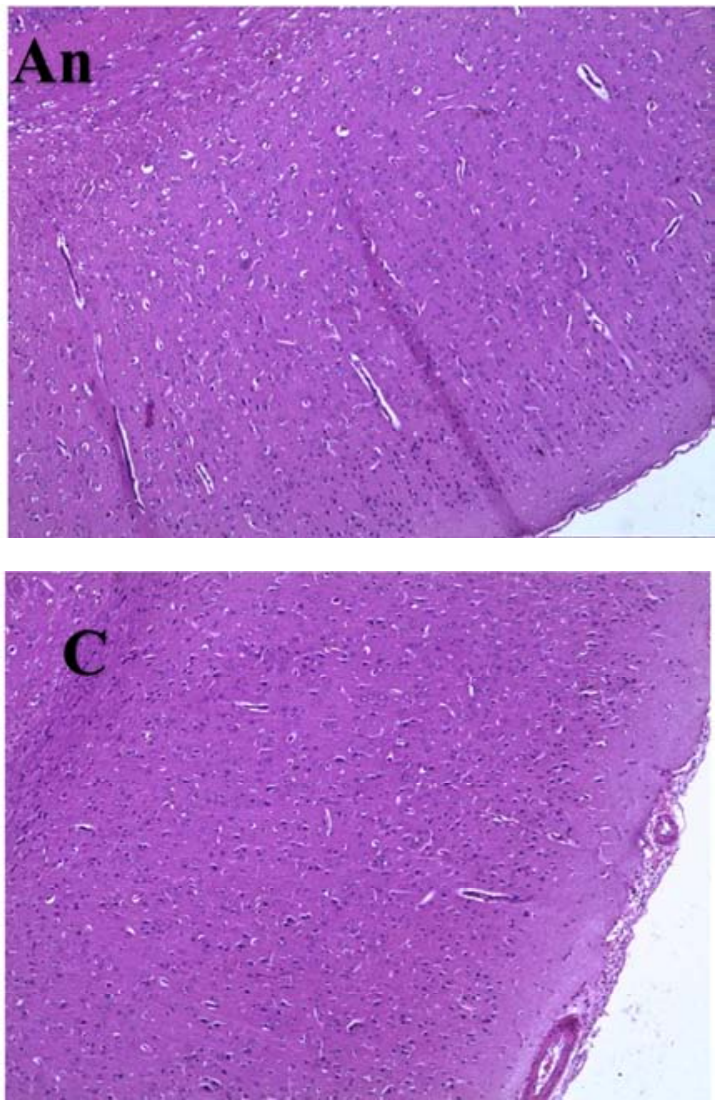

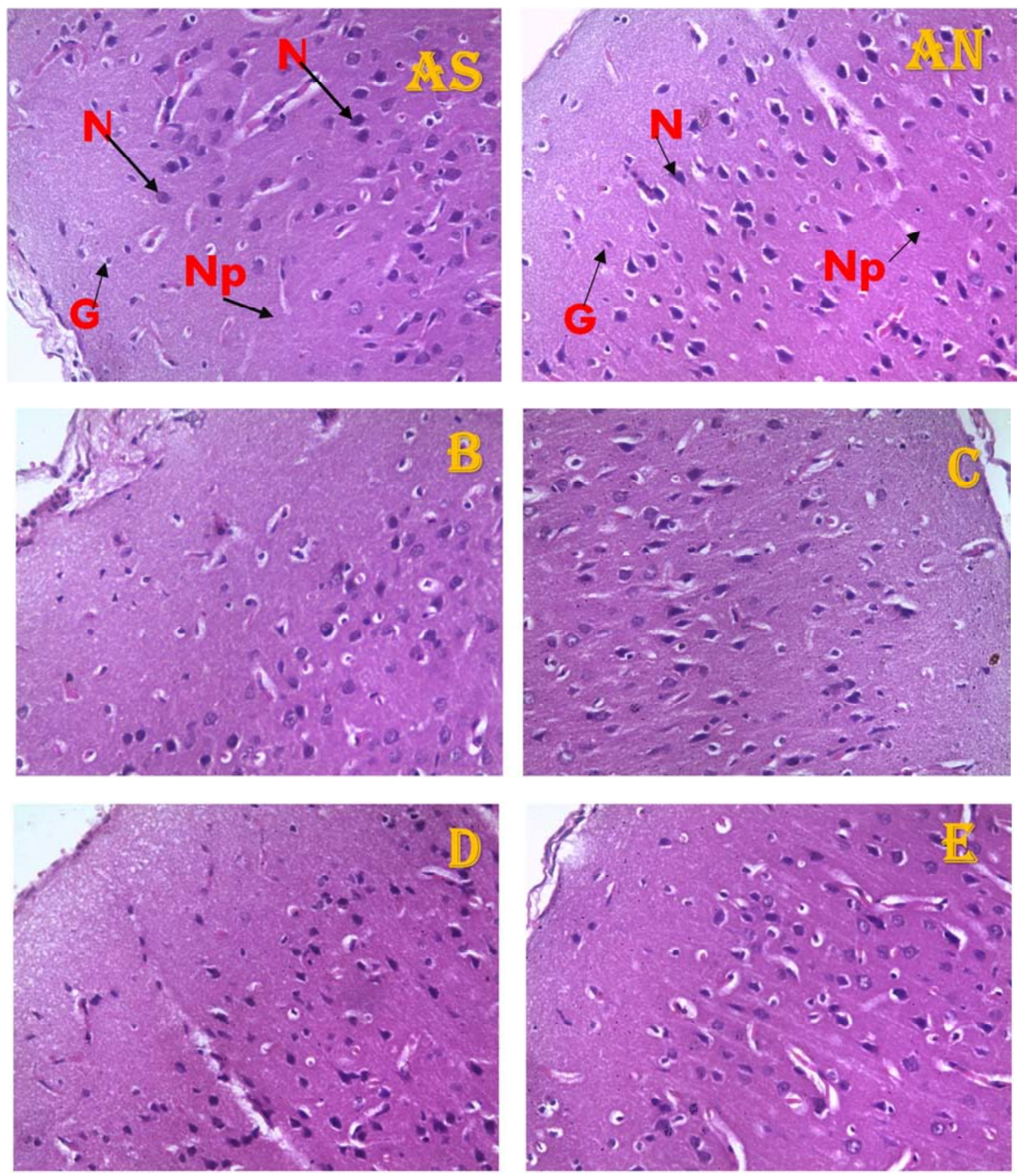

Figure 2. $H \&$ E stained prefrontal cortex (400X). As; group A (SHAM), An; group A (N-SHAM), B; group B (5MINS), C; group C (10MINS), D; group D (15MINS), E; group E (20MINS). N=Neuron, $G=$ Glial Cells, $N p=$ Neuropil.
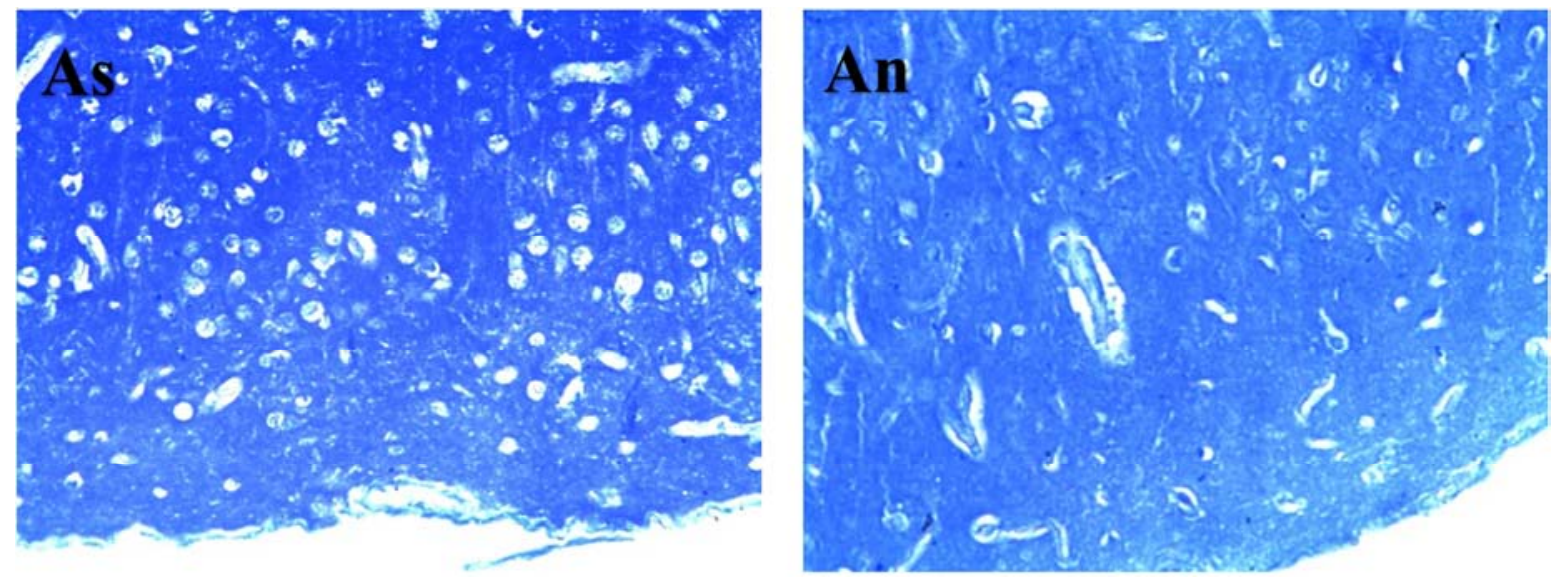

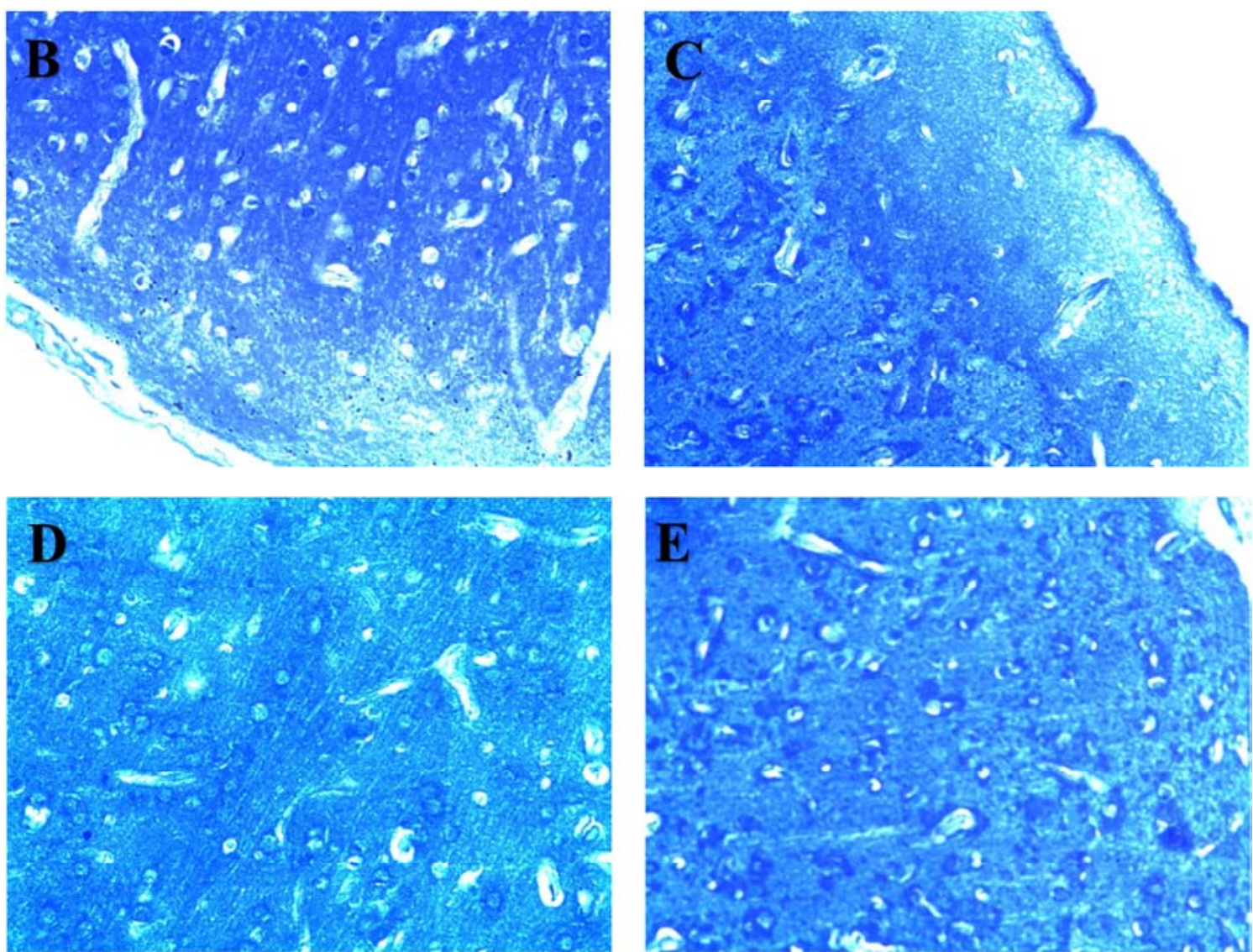

Figure 3. Photomicrographs of sections of the prefrontal cortex stained with Luxol Fast Blue to demonstrate the myelin sheath integrity. (400X). As; group A (SHAM), An; group A (N-SHAM), B; group B (5MINS), C; group C (10MINS), D; group D (15MINS), E; group E (20MINS).
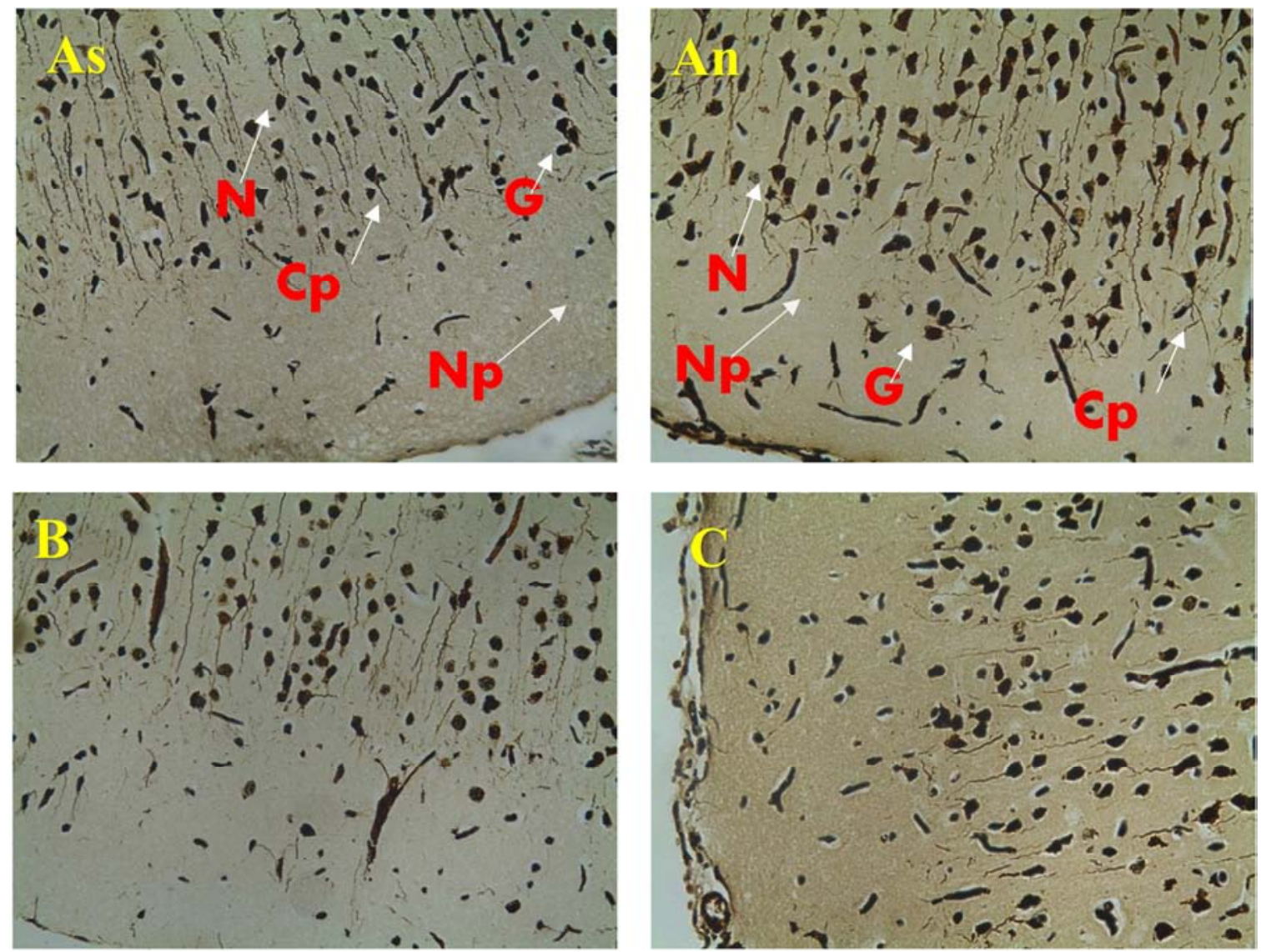

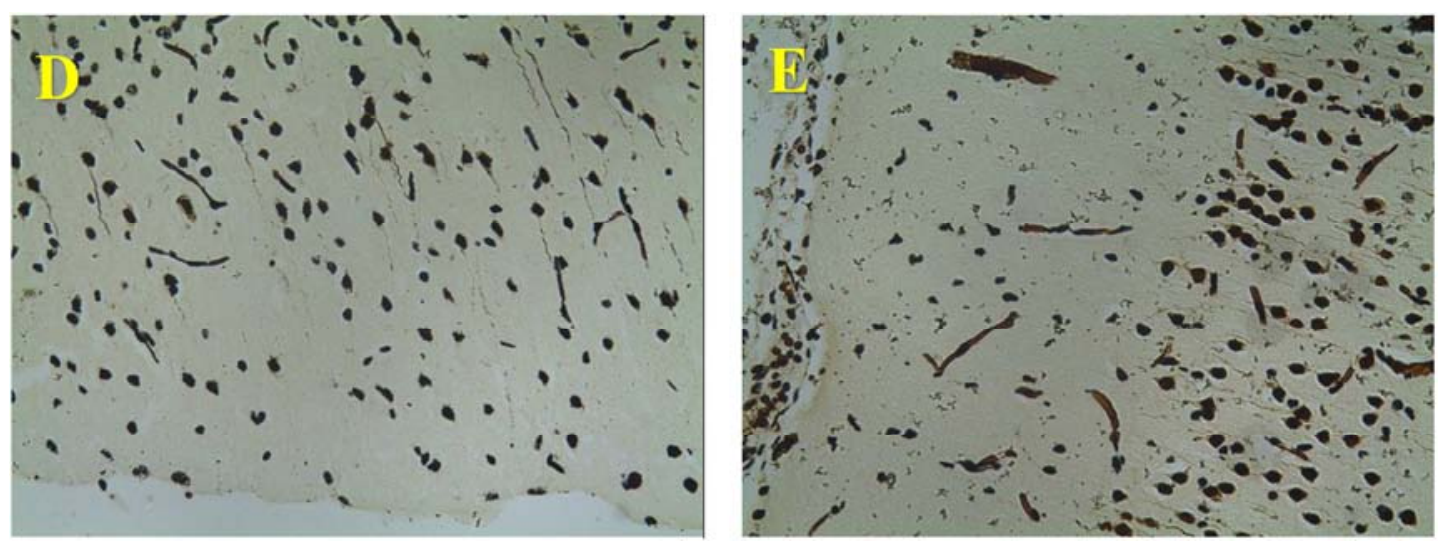

Figure 4. Photomicrographs of sections of the prefrontal cortex stained wih Bielchowsky silver impregnation to demonstrate the nerve fibers (Axons and Dendrites) (400X). As; group A (SHAM), An; group A (N-SHAM), B; group B (5MINS), C; group C (10MINS), D; group D (15MINS), E; group E (20MINS). $N=$ Neuron, $G=$ Glial Cells, $N p=$ Neuropil, $C p=$ Cell Processes/Neurites.

\section{HISTOMORPHOMETRIC RESULTS}

Figure 5 shows histomorphometric result of the neuronal cell count across all groups, no significant difference was observed in groups A [SHAM] [47.00 \pm 4.97$]$ and A [N-SHAM] [36.50 \pm 2.96$]$ when compared with the rest of the treated groups B [5MINS] [34.00 \pm 5.03 ], C [10MINS] [39.75 \pm 4.77$]$, D [15MINS] [39.75 \pm 4.77$],$ E [20MINS] [39.50 \pm 4.05$]$.

\section{NEURONAL CELL COUNT}

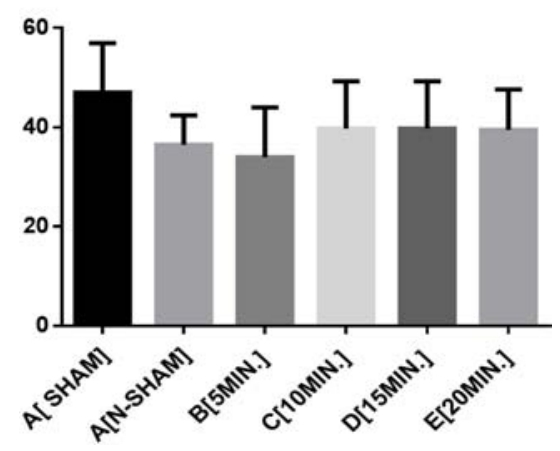

Figure 5. Neuronal cell count across all groups. Values are mean $\pm S E M$ of data obtained; $p$ values $(p<0.05)$. A (SHAM) (tDCS for 30 seconds), A (NSHAM) (no tDCS), B (5MINS) (tDCS for 5minutes), C (10MINS) (tDCS for 10minutes), D (15MINS) (tDCS for 15minutes), E (20MINS) ( $t D C S$ for 20minutes).

\section{GLIA CELL COUNT}

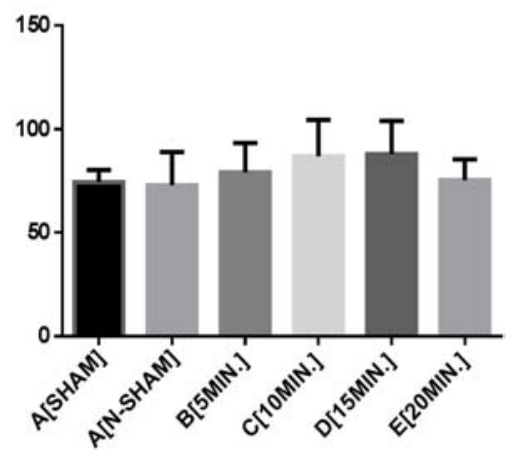

Figure 6. Glia cell count across all groups. Values are mean \pm SEM of data obtained; $p$ values $(p<0.05)$. A (SHAM) (tDCS for 30 seconds), A (N-SHAM) (no tDCS), B (5MINS) (tDCS for 5minutes), $C$ (10MINS) (tDCS for 10minutes), D (15MINS) (tDCS for 15minutes), E (20MINS) (tDCS for 20minutes).
Figure 6 shows histomorphometric result of the glia cell count across all groups, there was no observable significant difference in groups A [SHAM] [74.50 2.96$]$ and A [NSHAM] [73.00 \pm 8.01$]$ when compared with the rest of the treated groups B [5MINS] [79.25 \pm 7.01$], \quad \mathrm{C}$ [10MINS]

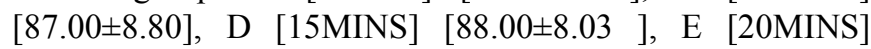
$[75.50 \pm 5.06]$.

\section{Discussion}

Transcranial direct current stimulation of the DLPFC results in a shift in the excitability which occur during and after stimulation [10]. Research in enhancing working memory is definitely one of the major things that can be done with the use of the tDCS. Anodal tDCS applied on the DLPFC facilitate working memory processes [11]. Findings show that anodal tDCS over the right DLPFC may prevent working memory impairment induced by acute stress [12]. Stimulation of the primary motor cortex (M1) and dorsolateral prefrontal cortex (DLPFC) reduces the perception of pain. Researches show that anodal tDCS of the DLPFC could decrease the perception of unpleasantness and reduces emotional discomfort or pain.

There is dearth of literature on the effects of transcranial direct current stimulation on the cytoarchitecture of the brain and this was the specific purpose of the present study. The extensive prospective use of modernized tDCS in the treatment of various disease raises the concern on the effect of tDCS on the brain as there could be possible side effects that might be worse than the treated disorder.

In the present study, as shown in figures 1-6 there were no significant differences in the histology and histomorphometric analysis of the prefrontal cortex structures. The neurons and the glial cells where not morphological different across the treated groups when compared with group A (N-SHAM) which served as the morphological control and this may have been as a result of the duration, frequency or intensity of the stimulation.

Figure 2 showed the photomicrograph of the $\mathrm{H}$ \& $\mathrm{E}$ stained prefrontal cortex at 400X. Group A showed a well demostrated neurons with its prominent cell body and darkly 
stained nuclues which is centrally placed and clearly observable cytoplasm with a clearly obsevable dendrites projecting out of the cell body and the neuroglias are well demostrated across the photomicrographs. These features in group A was also observed across the rest of the treated groups without any feaures of prominent eosinophilic cytoplasm or neuronal swelling (which are signs of neuronal damage) within the cytoplasm of the treated groups.

The Luxol fast blue (LFB) is a commonly used stain for the integrity of the myelin sheath or to detect demyelination in the central nervous system [18]. In the present study, Figure 3 showed the photomicrographs section stained with LFB. Photomicrograph slides of Group A showed a well define myelin sheath fibers and these ware also observed in the rest of the treated group without any form of demyelination, which is an indication that tDCS does not affect the integrity of the myelin sheath.

Bielschowsky technique is a silver staining method used to demonstrate neurites (axons, dendrites), nerve fibers and neurofibrillary tangles [19] in the present study, as shown in Figure 4, the photomicrographs of the control group showed well demonstrated neurons with clearly observable cell processes which were also observed in the rest of the treated groups. The cytoarchitectural findings from the presents study suggest the neuroprotective or neuro-enhancing effects of the tDCS on the brain rather than neurodegeneration and this can be corroborated with the works of Maria [20] who worked on the effects of tDCS on the hippocampus and reported an increase in hippocampal long term potentiation (LTP) and learning and memory following Anodal tDCS. Her results also corroborate the positive effects of tDCS on the brain structure and function that tDCS enhances rather than serving as neurodegenerative. LTP is a persistent strengthening of the synapses that is based on recent pattern of activity which produces a very long lasting increase in transmission of impulses in between two neurons and this is considered as one of the major cellular mechanism that underlines learning and memory $[21,22]$. Findings from the present study was also supported by the earlier report [23] that investigated that tDCS effects on repetitive mild traumatic brain injury in rats brain and reported therapeutic benefits of the tDCS on loss of consciousness and electrophysiological changes such as induced neural plasticity and early recovery of loss of consciousness. This would not have been possible if tDCS had neurodegenerative effects on the neurons and supporting cells of the brain. Based on the result shown in figures 1-4 and the histomorphometric analysis in figures 5 and 6 , it is evident that short term transcranial direct current stimulation of the brain have no significant effect on the histomorphology of the brain.

\section{Conclusion}

In conclusion, the short term repeated use of tDCS do not have any neurodegenerative effect making it safe for use in the enhancement of the effectiveness of the brain and also in the treatment of Parkinson's disease, Alzheimer's, depression, anxiety, memory loss, neuropathic pain among others. This research only demonstrates the short term of tDCS on the brain, so the possibility of the transcranial direct current stimulation causing neural degeneration over a long period of time should be further investigated.

\section{References}

[1] Brunoni Andre, Nitsche Micheal, Loo Colleen. Transcranial direct current stimulation in Neuropsychiatric Disorders: Clinical Principles and Management. Chapter 7: Neurocognitive effects of tDCS in the healthy brain. Springer International Publishing Switzerland. (2016) 103-110.

[2] De Aguiar V. TDCS in post-stroke aphasia: the role of stimulation parameters, behavioral treatment and patient characteristics. Cortex; 63 (2015) 296-16.

[3] Bennabi Djamila, Solene pedron, and Vincent Van Waes (2014). Transcranial direct current stimulation for memory enhancement: from clinical research to animal models. Front Syst Neuroscience. 8: (2014) 159.

[4] Badre, D., and Wagner, A. D. (2004). Selection, integration, and conflict monitoring: Assessing the nature and generality of prefrontal cognitive control mechanisms. Neuron, 41, 473487.

[5] Kobayashi S. Reward Neurophysiology and Primate Cerebral cortex. Encyclopedia of Neuroscience (2009).

[6] Pierrot-Deseillingny Ch, Muri RM, Nyffeler T, Milea D. The role of the human dorsolateral prefrontal cortex in ocular motor behavior. Ann N Y Acad Sci; 1039: (2005) 239-51.

[7] Jones EG. Viewpoint: the core and matrix of thalamic organization. Neuroscience 85 (1998): 331-45.

[8] Goldman-Rakic, Patricia S. Architecture of the prefrontal cortex and the central executive. Annals of the New York Academy of Sciences 769 (1995) 71-83.

[9] D Badre, AD Wagner Left ventrolateral prefrontal cortex and the cognitive control of memory Neuropsychologia 45 (2007), 2883-2901.

[10] Nitsche M. A, Cohen Leonardo G, Wassermann Eric M, Priori Alberto, Lang Nicolas, Antal Andrea, Paulus Walter, Hummel Friedhelm, Boggio Paulo S, Fregni Felipe, Pascual-Leone Alvaro. Transcranial direct current stimulation: state of the art. Brain Stimulation. 1 (2008). (3): 206-23.

[11] Fregni F., Boggio PS, Nitsche M., Bermpohl F, Antal A, Feredoes E, et al. Anodal transcranial direct current stimulation of prefrontal cortex enhances working memory. Experimental brain research. Experimentelle Hirnforschung. Exper Cerebral; 166 (2005) 23-0.

[12] Mario Bogdanov, Christian C. Ruff and Lars Schwabe. Transcranial stimulation over the dorsolateral prefrontal cortex increases the impact of past expenses on decisionmaking. Cerebral cortex, (2015) 1-9.

[13] Lauren Naomi Spezia Adachi, Wolnei Caumo, Gabriela Laste, Liciane Fernande Medeiros, Joanna Ripoll Rozisky, Andressa De Souza, Felipe Fregni, Iraci L. S. Torres. Reversal of chronic stress-induced pain by transcranial direct current stimulation (tDCS) in an animal model. Brain Research. Vol. 1489: (2012) 17-26. 
[14] Nitsche M. A, Paulus W. Sustained excitability elevations by transcranial DC motor cortex stimulation in humans. Neurology; 57 (2001): 1899-901.

[15] Tanefumi B, Masahiro K, Takao Y, Takamasa M, Akihiko K, Tetsuro S, et al. Electrical Stimulation of the Cerebral Cortex Exerts Antiapoptotic, Angiogenic and Anti-Inflammatory Effects in Ischemic Stroke Rats Through Phosphoinositide 3Kinase/Akt Signaling Pathway. Am Heart Associ; 10 (2009) 563-98.

[16] Takano Y, Yokawac T, Masudac A, Niimic J, Tanakad S, Hironakaa. A rat model for measuring the effectiveness of transcranial direct current stimulation using fMRI, Neuroscience Letter 491: (2011) 40-43.

[17] Monai H, Ohkura M, Tanaka M, Yuki O, Konno A, Hirai H, et al. Calcium imaging reveals glial involvement in transcranial direct current stimulation-induced plasticity in mouse brain. Natur Comm 2016; 7: 1-10. Moreraft RJ, Yterin EH, Ramchandaran VS (2002). PFC: prefrontal cortex. Encyclopedia of human brain, USA: Academic Press; (2002) 11-26.

[18] Kluver H., Barrera E. "A method for the combined staining of cells and fibers in the Nervous system". J. Neuropath. Exp. Neurol. 12 (1953) 400-403.
[19] Erickson-Davis CR, Faust PL, Vonsattel JP, Gupta S, Honig LS, Louis ED. "'Hairy baskets" associated with degenerative Purkinje cell changes in essential tremor" J. Neuropathol. Exp. Neurol. 69 (2010): 262-71.

[20] Maria Vittoria Podda1,*, Sara Cocco1,*, Alessia Mastrodonato1, Salvatore Fusco1, Lucia Leone1, Saviana Antonella Barbati1, Claudia Colussi 1, 2, Cristian Ripoli1 \& Claudio Grassi1, 3 Anodal transcranial direct current stimulation boosts synaptic plasticity and memory in mice via epigenetic regulation of Bdnf expression.

[21] Paradiso, Michael A.; Bear, Mark F.; Connors, Barry W. Neuroscience: Exploring the Brain. Hagerstwon, MD: Lippincott Williams \& Wilkins (2007) p. 718. ISBN 0-78176003-8.

[22] Bliss TV, Collingridge GL. "A synaptic model of memory: long-term potentiation in the hippocampus". Nature. 361 (1993): 31-39.

[23] Ho Jeong Kim and Soo Jeong Han. Anodal Transcranial Direct Current Stimulation Provokes Neuroplasticity in Repetitive Mild Traumatic Brain Injury in Rats. Hindawi Neural Plasticity. Article ID 1372946, (2017). 